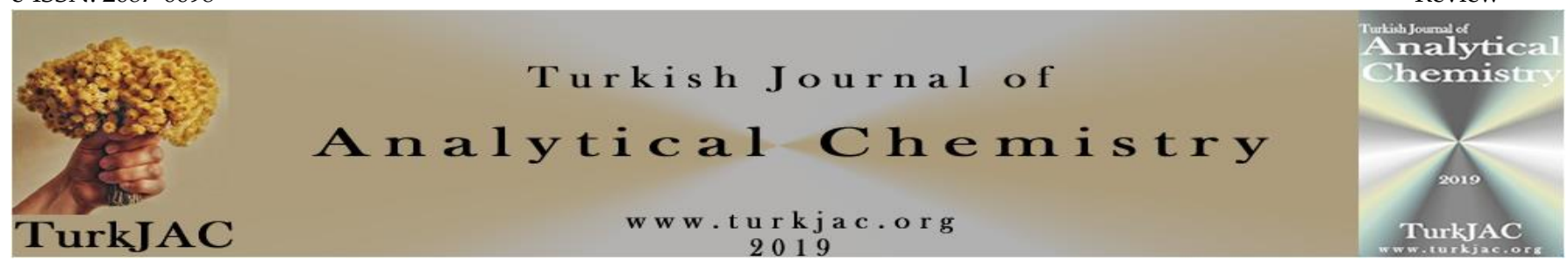

\title{
Bioactivities of Anacyclus pyrethrum (L.) Lag. extracts and natural products
}

\author{
Saravanan Vivekanandarajah Sathasivampillai ${ }^{*}$ (D), Sujarajini Varatharasan² (D) \\ ${ }^{1}$ KnowledgeLink Group, Inc., Waltham, MA 02451, USA \\ ${ }^{2}$ South Eastern University of Sri Lanka, Department of Biological Sciences, Sammanthurai, Sri Lanka
}

\begin{abstract}
Anacyclus pyrethrum (L.) Lag. is an herb that fits into the Asteraceae family. It has been using to treat several disorders include lung infections, liver diseases, nervous system diseases, and rheumatism in ethnomedicines. Compounds including pellitorine, anacycline, spilanthol, pyracyclumine A, and agrocybenine have been isolated from this plant species. Thus, this overview work intends to scrutinize, sum up, and record the available scientific evidence of bioactivity of A. pyrethrum. This work would be very convenient for future bioactivity and phytochemical studies of this plant species. Electronic databases such as the Web of Science, Scopus, ScienceDirect, and PubMed were applied to identify appropriate published articles associated with bioactivities of A. pyrethrum from 1900 to November 2020. Until now, scientific evidence of bioactivity for various parts of this plant species are existing in clinical, in vivo, and in vitro studies. Bioactivities are including anesthetic, anti-depressant, anti-epileptic, anticonvulsive, and blood circulatory activities in diverse assays and models. Seven antiprotozoal active compounds and an anti-inflammatory active compound have been identified in A. pyrethrum. More bioactivities and phytochemical linked researches should be conducted to generate more scientific evidence for the ethnomedicinal uses. This work scrutinized, summed up, and recorded the currently available scientific evidence of bioactivity of A. pyrethrum.
\end{abstract}

Keywords: Anacyclus pyrethrum, Asteraceae, Sri Lanka, Siddha Medicine, bioactivities

\section{Introduction}

Anacyclus pyrethrum (L.) Lag. is an herb that fits into the Asteraceae family. It is native to Spain, Morocco, and Algeria and it has been introduced into Sri Lanka, India, Ukraine, Germany, Myanmar, France, and Poland [1]. A. pyrethrum has been using to treat several disorders include lung infections, liver diseases, nervous system diseases, sciatica, fever, malaria, paralysis, epilepsy, sore throat, rheumatism, colds, neuralgia, toothache, sleep apnea, diaphoresis, poor blood circulation, salivary gland illnesses, head catarrh, nostril catarrh, and urinary tract infections in ethnomedicines [2-12]. It is also utilized for revitalization and vivacity in Ayurveda [11,13]. A. pyrethrum is called Akkarahaaram (அக்கரகாரம்) in Tamil and its root are applied to prepare antidiabetic preparations in Sri Lankan Siddha Medicine [14,15]. Compounds including squalene, stigmasterol, $\gamma$ stigmasterol, octadecanoic acid, palmitic acid, pellitorine, N-propylnona-2,5-dienamide, anacycline, N-Methyanacycline, dehydro-anacycline, dehydromatricaric acid, spilanthol, pyracyclumine $\mathrm{A}$, pyracyclumine $B$, pyracyclumine $C$, pyracyclumine $D$, pyracyclumine $\mathrm{E}$, pyracyclumine $\mathrm{F}$, pyracyclumine $\mathrm{G}$, pyracyclumine $\mathrm{H}$, dodeca-2E,4E-dienoic acid 4Citation: Saravanan Vivekanandarajah Sathasivampillai, Sujarajini Varatharasan, Bioactivities of Anacyclus pyrethrum (L.) Lag. extracts and natural products, Turk J Anal Chem, 2(2), 2020, 55-61.

ot) https://doi.org/ hydroxyphenylethylamide, pyracyclumine I, pyracyclumine J, deca-2E,4E-dienoic acid 4hydroxyphenylethylamide, tetradeca-2E,4E-dienoic acid 4-hydroxyphenylethyl amide, undeca-2E,4E-diene-8,10diynoic acid 2-phenylethyl amide, and agrocybenine have been isolated from this plant species using analytical chemistry techniques like liquid chromatography-mass spectrometry (LC-MS), gas chromatography-mass spectrometry (GC-MS), nuclear magnetic resonance spectroscopy (NMR), ultravioletvisible (UV-Vis.) spectroscopy, and infrared (IR) spectroscopy [16-22].

\section{Aims and objectives}

Thus far, there is no systematic comprehensive review of bioactivities of $A$. pyrethrum. Thus, this overview work intends to scrutinize, sum up, and record the available bioactive scientific evidence of A. pyrethrum. This work would be very convenient for future bioactivity and phytochemical studies of this plant species.

*Author of correspondence: vivekanandarajahs@yahoo.co.uk Phone: +94778832430

Received: November 24, 2020

Accepted: December 21, 2020 


\section{Materials and methods}

Electronic databases the Web of Science, Scopus, ScienceDirect, and PubMed were applied to identify appropriate published articles related to bioactivities of A. pyrethrum from 1900 to November 2020. The databases used in this review work have greater advantages than Google Scholar. These databases contain multiple organizing functions like categorizing the document type, subject, country, affiliation, and year. Anyhow, not all the abstracts, full articles, and journals obtained from Google Scholar search results are available at the moment for analysis. "Anacyclus pyrethrum" (in double quotation marks) was employed as an exploration term and the results were limited to subjects like Pharmacology, Toxicology and Pharmaceutics, Medicine, Biochemistry, Genetics and Molecular Biology, Chemistry, Agricultural and Biological Sciences, and Multidisciplinary and then the reviews were excluded from the document type.

\section{Results and discussions}

\subsection{Bioactivities of A. pyrethrum}

Search results obtained from the literature review are presented in Table 1. Until now, scientific evidence related to the bioactivity of various parts of this plant species exists for clinical, in vivo, and in vitro studies. However, most of them are in vivo studies. Anesthetic $[23,24]$, antibacterial [17,21,25], anticancer [26], antidementia [27], antidiabetic [28,29], antifungal [21], anti-inflammatory [20,30,31], antilipidemic [28], antimutagenic [32], antioxidant [28,30,33-35], antiprotozoal [36], blood circulatory [37], oxidative DNA damage preventive [33], anabolic [38], androgenic [39], anticonvulsive [30,40-43], antidepressant [44], antiepileptic [45], aphrodisiac [38,46], fertility improvement [47], hepatoprotective [48], immunomodulatory [4,49,50], neuroprotective [30], reproductive tract [38], and spermatogenic [39] activities in diverse assays and models are available. Anyhow, the majority of the scientific evidence is existing for antioxidant activities and roots revealed a greater number of bioactivities. Further, water has been used in the majority of the studies to prepare the extracts. Seven bioactive compounds such 9,10-dehydropellitorine, deca-2E,4Edienoic acid 2-phenylethylamide, deca-2E,4E-dienoic acid tyramide, dodeca-2E,4E-dienoic acid 4-hydroxy-2phenylethylamide, pellitorine, tetradeca-2E,4E,12Ztrien-8,10-diynoic acid isobutylamide, and undeca2E,4E-dien-8,10-diynoic acid isopentylamide have been identified in A. pyrethrum. Except for deca-2E,4Edienoic acid tyramide all other compounds have shown antiprotozoal activities in a variety of in vitro assays [36].
Whereas deca-2E,4E-dienoic acid tyramide has been unveiled anti-inflammatory activity [20]. Pellitorine has exhibited both blood circulatory and antiprotozoal activities [36,37]. Hitherto, exclusively scientific evidence is existing for liver diseases, nervous system diseases, sciatica, epilepsy, rheumatism, neuralgia, sleep apnea, and poor blood circulation remedies. Significant studies based on the available lowest concentration/dose of treatment are conferred underneath.

\subsection{Clinical trial}

\subsubsection{Anesthetic activity}

A solution containing $2 \%$ of root alcohol extract (freshly dissolved in sterile distilled water) was administered to 200 dental patients and it exhibited anesthetic activity in extended oral reconstructive surgeries. The extract did not show any side effect and seemed safer at a lower dose. Xylocaine was used as a positive control in this clinical trial [24].

\subsection{In vivo studies}

\subsubsection{Androgenic activity}

A dose of $50 \mathrm{mg} / \mathrm{kg}$ of root aqueous and ethanolic extracts was orally administered to rats and noticeable anabolic effects in two different studies were observed $[38,39]$.

\subsubsection{Anticonvulsive activity}

Root ethanolic extract $(200 \mathrm{mg} / \mathrm{kg})$ was orally administered to mice and it showed anticonvulsive properties by curative effects in maximal electroshock seizures [41].

\subsubsection{Antidepressant activity}

Badhe et al. (2010) studied the antidepressant effects of root aqueous extract $(50 \mathrm{mg} / \mathrm{kg})$ in both clonidineinduced hypothermia and reserpine-induced hypothermia mice [44]. In this study, they performed various methods like haloperidol-induced catalepsy, locomotor activity, tail suspension test, and forced swim test. It has been observed that there was a rise in ambulatory behavior representing a stimulant outcome and increased movability.

\subsubsection{Antiepileptic activity}

Root hydroalcoholic extract at doses of 250 and 500 $\mathrm{mg} / \mathrm{kg}$ was injected into pentylenetetrazole-induced kindling mice and it protected cognitive diminishing by reducing oxidative stress [45].

\subsubsection{Anti-inflammatory activity}

Extracts prepared from roots $(3 \mathrm{mg})$ in water, chloroform, and ethanol were topically applied separately on arachidonic acid-induced ear edema in mice inhibited the inflammation. Indomethacin was used as a positive control in this research [31]. 
Table 1. Reported bioactivities of A. pyrethrum

\begin{tabular}{|c|c|c|c|c|c|c|}
\hline $\begin{array}{l}\text { Level of } \\
\text { scientific } \\
\text { evidence }\end{array}$ & Bioactivity & $\begin{array}{l}\text { Part } \\
\text { used }\end{array}$ & Extract / compound & Assay / model / subject & Dose / concentration & Ref. \\
\hline Clinical & Anesthetic & Root & Alcohol & Dental patient & $\begin{array}{l}2 \% \text { freshly dissolved in sterile } \\
\text { distilled water }\end{array}$ & [24] \\
\hline \multirow[t]{2}{*}{ In vivo } & Anesthetic & Root & Aqueous & Guinea pig & $1 \%$ & [23] \\
\hline & & Root & Ethanol & Guinea pig & $2 \%$ & \\
\hline In vivo & Androgenic & Root & Aqueous, ethanol & Rat & $50 \mathrm{mg} / \mathrm{kg}$ & {$[38,39]$} \\
\hline In vivo & Androgenic & Root & Ethanol & Rat & $100 \mathrm{mg} / \mathrm{kg}$ & {$[47]$} \\
\hline In vivo & Anticonvulsive & Root & Ethanol & Pilocarpine-induced epilepsy & $200,400 \mathrm{mg} / \mathrm{kg}$ & [40] \\
\hline In vivo & Anticonvulsive & Root & Ethanol & Maximal electroshock seizure & $200,400,600 \mathrm{mg} / \mathrm{kg}$ & [41] \\
\hline In vivo & Anticonvulsive & Root & Methanol (50\%) & Cognitive impairment & $250,500,1000 \mathrm{mg} / \mathrm{kg}$ & [42] \\
\hline In vivo & Anticonvulsive & Root & Aqueous, methanol & Kainic acid-induced-status epilepticus & $5 \mathrm{~g} / \mathrm{L}$ & [43] \\
\hline In vivo & Antidepressant & Root & Aqueous & $\begin{array}{l}\text { Clonidine-induced hypothermia, reserpine- } \\
\text { induced hypothermia }\end{array}$ & $50,100,200 \mathrm{mg} / \mathrm{kg}$ & [44] \\
\hline In vivo & Antiepileptic & Root & Hydroalcoholic & Pentylenetetrazole-induced kindling & $250,500 \mathrm{mg} / \mathrm{kg}$ & [45] \\
\hline In vivo & Anti-inflammatory & Root & Aqueous, methanol & $\begin{array}{l}\text { Complete Freund's Adjuvant-induced paw } \\
\text { edema, xylene-induced ear edema }\end{array}$ & $125 \mathrm{mg} / \mathrm{kg}$ & [30] \\
\hline \multirow[t]{3}{*}{ In vivo } & Anti-inflammatory & Root & Aqueous & Arachidonic acid-induced ear oedema & $3 \mathrm{mg}$ & {$[31]$} \\
\hline & & Root & Aqueous, chloroform, ethanol & Carrageenan-induced sub plantar oedema & $100 \mathrm{mg} / \mathrm{kg}$ & \\
\hline & & Root & Chloroform, ethanol & Arachidonic acid-induced ear oedema & $3 \mathrm{mg}$ & \\
\hline In vivo & Aphrodisiac & Root & Petroleum ether & Rat & $50,100 \mathrm{mg} / \mathrm{kg}$ & [46] \\
\hline In vivo & Blood circulatory & Root & Pellitorine & Rat & $5 \mathrm{mg} / \mathrm{mL}$ & [37] \\
\hline In vivo & Antihepatotoxic & Root & Ethanol (50\%) & Antitubercular drug-induced hepatotoxic & $200,400 \mathrm{mg} / \mathrm{kg}$ & [48] \\
\hline In vivo & Immunomodulatory & Root & Aqueous & Mouse & $10 \mathrm{mg} / \mathrm{kg}$ & [4] \\
\hline In vivo & Immunomodulatory & Root & Petroleum ether & $\begin{array}{l}\text { Cyclophosphamide-induced } \\
\text { Immunosuppression }\end{array}$ & $50,100 \mathrm{mg} / \mathrm{kg}$ & [50] \\
\hline In vivo & Immunomodulatory & Root & Methanol & Rat & $50,100,200 \mathrm{mg} / \mathrm{kg}$ & [49] \\
\hline In vivo & Antineurotoxic & Root & Aqueous, methanol & Kainic acid-induced-status epilepticus & $5 \mathrm{~g} / \mathrm{L}$ & [43] \\
\hline In vitro & Antibacterial & Root & Ethanol & $\begin{array}{l}\text { Bacillus subtilis, Enterobacter aerogenes, } \\
\text { Enterecoccus faecalis, Enterococcus } \\
\text { durans, Escherichia coli, Klebsiella } \\
\text { pneumoniae, Listeria innocua, Listeria } \\
\text { monocytogenes, Pseudomonas aeruginosa, } \\
\text { Pseudomonas fluorescens, Salmonella } \\
\text { enteritidis, Salmonella infantis, } \\
\text { Staphylococcus aureus, Staphylococcus } \\
\text { epidermidis }\end{array}$ & NS & [17] \\
\hline In vitro & Antibacterial & Root & Methanol & Escherichia coli & $\begin{array}{l}1000 \mathrm{mg} / \mathrm{mL}(\mathrm{MIC}), 800 \\
\mathrm{mg} / \mathrm{mL}(\mathrm{MBC})\end{array}$ & [25] \\
\hline In vitro & Antibacterial & Aerial & Essential oil & Staphylococcus aureus & $1.25 \mathrm{mg} / \mathrm{mL}$ & [21] \\
\hline In vitro & Anticancer & Aerial & Ethanol & Human colorectal cancer cell & $\begin{array}{l}64.75 \mu \mathrm{g} / \mathrm{mL}\left(\mathrm{IC}_{50}\right) \text { for } 24 \mathrm{~h} \\
105.9 \mu \mathrm{g} / \mathrm{mL}\left(\mathrm{IC}_{50}\right) \text { for } 48 \mathrm{~h}\end{array}$ & [26] \\
\hline \multirow[t]{2}{*}{ In vitro } & Antidementia & Root & Chloroform & Acetylcholinesterase inhibitory & $150 \mathrm{mg} / \mathrm{mL}\left(\mathrm{IC}_{50}\right)$ & [27] \\
\hline & & Root & Ethanol & Acetylcholinesterase inhibitory & $70 \mathrm{mg} / \mathrm{mL}\left(\mathrm{IC}_{50}\right)$ & \\
\hline In vitro & Antidiabetic & NS & Aqueous & $\alpha$-Amylase inhibitory & $39.1 \mu \mathrm{g} / \mathrm{mL}\left(\mathrm{IC}_{50}\right)$ & [28] \\
\hline \multirow[t]{5}{*}{ In vitro } & Antidiabetic & Root & Acetone & $\alpha$-Amylase inhibitory & $57.29 \mu \mathrm{g} / \mathrm{mL}\left(\mathrm{IC}_{50}\right)$ & [29] \\
\hline & & Root & Aqueous & $\alpha$-Amylase inhibitory & $49.36 \mu \mathrm{g} / \mathrm{mL}\left(\mathrm{IC}_{50}\right)$ & \\
\hline & & Root & Chloroform & $\alpha$-Amylase inhibitory & $40.34 \mu \mathrm{g} / \mathrm{mL}\left(\mathrm{IC}_{50}\right)$ & \\
\hline & & Root & Ethanol & $\alpha$-Amylase inhibitory & $29.25 \mu \mathrm{g} / \mathrm{mL}\left(\mathrm{IC}_{50}\right)$ & \\
\hline & & Root & Ethyl acetate & $\alpha$-Amylase inhibitory & $52.52 \mu \mathrm{g} / \mathrm{mL}\left(\mathrm{IC}_{50}\right)$ & \\
\hline In vitro & Antifungal & Root & Ethanol & Candida albicans & NS & [17] \\
\hline In vitro & Antifungal & Aerial & Essential oil & Candida albicans & $0.72 \mathrm{mg} / \mathrm{mL}$ & [21] \\
\hline \multirow[t]{2}{*}{ In vitro } & Anti-inflammatory & Root & $\begin{array}{l}\text { Deca-2E,4E-dienoic acid } \\
\text { tyramide }\end{array}$ & $\begin{array}{l}\text { 5-lipoxygenase inhibitory, cyclooxygenase } \\
\text { inhibitory }\end{array}$ & $50 \mu \mathrm{g} / \mathrm{mL}$ & [20] \\
\hline & & Root & Hexane & $\begin{array}{l}\text { 5-lipoxygenase inhibitory, cyclooxygenase } \\
\text { inhibitory }\end{array}$ & $11.5 \mathrm{~g} / \mathrm{mL}$ & \\
\hline In vitro & Antilipidemic & NS & Aqueous & $\begin{array}{l}\text { Rat intestinal disaccharidases inhibitory } \\
\text { (Lipase) }\end{array}$ & NS & [28] \\
\hline In vitro & Antimutagenic & Root & Chloroform & Ames Salmonella / microsome & $1 \mathrm{mg} / \mathrm{plate}$ & [32] \\
\hline In vitro & Antioxidant & NS & Aqueous & ABTS radical scavenging & NS & [28] \\
\hline In vitro & Antioxidant & Root & Methanol (50\%) & ABTS radical scavenging & $31.76 \mu \mathrm{g} / \mathrm{mL}\left(\mathrm{IC}_{50}\right)$ & [33] \\
\hline
\end{tabular}




\begin{tabular}{|c|c|c|c|c|c|c|}
\hline $\begin{array}{l}\text { Level of } \\
\text { scientific } \\
\text { evidence }\end{array}$ & Bioactivity & $\begin{array}{l}\text { Part } \\
\text { used }\end{array}$ & Extract / compound & Assay / model / subject & Dose / concentration & Ref. \\
\hline & & Root & Methanol (50\%) & DPPH radical scavenging & $467.1 \mu \mathrm{g} / \mathrm{mL}\left(\mathrm{IC}_{50}\right)$ & \\
\hline & & Root & Methanol (50\%) & Peroxynitrite scavenging & $1.13 \mu \mathrm{g} / \mathrm{mL}\left(\mathrm{IC}_{50}\right)$ & \\
\hline & & Root & Methanol (50\%) & $\mathrm{OH}$ radical scavenging & $41.22 \mu \mathrm{g} / \mathrm{mL}\left(\mathrm{IC}_{50}\right)$ & \\
\hline & & Root & Methanol (50\%) & Superoxide radical scavenging & $83.49 \mu \mathrm{g} / \mathrm{mL}\left(\mathrm{IC}_{50}\right)$ & \\
\hline In vitro & Antioxidant & Root & Methanol & DPPH radical scavenging, FRAP & NS & {$[34]$} \\
\hline In vitro & Antioxidant & Seed & Ethanol (70\%) & DPPH radical scavenging & $0.5,1.0,5.0,10.0 \mathrm{mg} / \mathrm{mL}$ & {$[35]$} \\
\hline \multirow[t]{6}{*}{ In vitro } & Antioxidant & Root & Aqueous & DPPH radical scavenging & $13.41 \mu \mathrm{g} / \mathrm{mL}\left(\mathrm{IC}_{50}\right)$ & {$[30]$} \\
\hline & & Root & Aqueous & FRAP & $60.17 \mu \mathrm{g} / \mathrm{mL}\left(\mathrm{IC}_{50}\right)$ & \\
\hline & & Root & Aqueous & $\beta$-carotene-linoleic acid bleaching & $120.66 \mu \mathrm{g} / \mathrm{mL}\left(\mathrm{IC}_{50}\right)$ & \\
\hline & & Root & Methanol & DPPH radical scavenging & $12.38 \mu \mathrm{g} / \mathrm{mL}\left(\mathrm{IC}_{50}\right)$ & \\
\hline & & Root & Methanol & FRAP & $50.89 \mu \mathrm{g} / \mathrm{mL}\left(\mathrm{IC}_{50}\right)$ & \\
\hline & & Root & Methanol & $\beta$-carotene-linoleic acid bleaching & $107.07 \mu \mathrm{g} / \mathrm{mL}\left(\mathrm{IC}_{50}\right)$ & \\
\hline \multirow[t]{24}{*}{ In vitro } & Antiprotozoal & Root & 9,10-dehydropellitorine & Leishmania donovani & $21.6 \mu \mathrm{M}\left(\mathrm{IC}_{50}\right)$ & [36] \\
\hline & & Root & 9,10-dehydropellitorine & Plasmodium falciparum & $32.2 \mu \mathrm{M}\left(\mathrm{IC}_{50}\right)$ & \\
\hline & & Root & 9,10-dehydropellitorine & Trypanosoma brucei rhodesiense & $13.2 \mu \mathrm{M}\left(\mathrm{IC}_{50}\right)$ & \\
\hline & & Root & 9,10-dehydropellitorine & Trypanosoma cruzi & $181 \mu \mathrm{M}\left(\mathrm{IC}_{50}\right)$ & \\
\hline & & Root & $\begin{array}{l}\text { Deca-2E,4E-dienoic acid 2- } \\
\text { phenylethylamide }\end{array}$ & Leishmania donovani & $11 \mu \mathrm{M}\left(\mathrm{IC}_{50}\right)$ & \\
\hline & & Root & $\begin{array}{l}\text { Deca-2E,4E-dienoic acid 2- } \\
\text { phenylethylamide }\end{array}$ & Plasmodium falciparum & $18 \mu \mathrm{M}\left(\mathrm{IC}_{50}\right)$ & \\
\hline & & Root & $\begin{array}{l}\text { Deca-2E,4E-dienoic acid 2- } \\
\text { phenylethylamide }\end{array}$ & Trypanosoma brucei rhodesiense & $14.9 \mu \mathrm{M}\left(\mathrm{IC}_{50}\right)$ & \\
\hline & & Root & $\begin{array}{l}\text { Deca-2E,4E-dienoic acid 2- } \\
\text { phenylethylamide }\end{array}$ & Trypanosoma cruzi & $18.5 \mu \mathrm{M}\left(\mathrm{IC}_{50}\right)$ & \\
\hline & & Root & Dichloromethane & Leishmania donovani & $4.22 \mu \mathrm{g} / \mathrm{mL}\left(\mathrm{IC}_{50}\right)$ & \\
\hline & & Root & Dichloromethane & Plasmodium falciparum & $3.04 \mu \mathrm{g} / \mathrm{mL}\left(\mathrm{IC}_{50}\right)$ & \\
\hline & & Root & Dichloromethane & Trypanosoma brucei rhodesiense & $10 \mu \mathrm{g} / \mathrm{mL}\left(\mathrm{IC}_{50}\right)$ & \\
\hline & & Root & Dichloromethane & Trypanosoma cruzi & $8.83 \mu \mathrm{g} / \mathrm{mL}\left(\mathrm{IC}_{50}\right)$ & \\
\hline & & Root & $\begin{array}{l}\text { Dodeca-2E,4E-dienoic acid 4- } \\
\text { hydroxy-2-phenylethylamide }\end{array}$ & Leishmania donovani & $13.3 \mu \mathrm{M}\left(\mathrm{IC}_{50}\right)$ & \\
\hline & & Root & $\begin{array}{l}\text { Dodeca-2E,4E-dienoic acid 4- } \\
\text { hydroxy-2-phenylethylamide }\end{array}$ & Plasmodium falciparum & $10.1 \mu \mathrm{M}\left(\mathrm{IC}_{50}\right)$ & \\
\hline & & Root & $\begin{array}{l}\text { Dodeca-2E,4E-dienoic acid 4- } \\
\text { hydroxy-2-phenylehylamide }\end{array}$ & Trypanosoma brucei rhodesiense & $7.17 \mu \mathrm{M}\left(\mathrm{IC}_{50}\right)$ & \\
\hline & & Root & $\begin{array}{l}\text { Dodeca-2E,4E-dienoic acid 4- } \\
\text { hydroxy-2-phenylethylamide }\end{array}$ & Trypanosoma cruzi & $5.97 \mu \mathrm{M}\left(\mathrm{IC}_{50}\right)$ & \\
\hline & & Root & $\begin{array}{l}\text { Tetradeca-2E,4E,12Z-trien-8,10- } \\
\text { diynoic acid isobutylamide }\end{array}$ & Leishmania donovani & $18.7 \mu \mathrm{M}\left(\mathrm{IC}_{50}\right)$ & \\
\hline & & Root & $\begin{array}{l}\text { Tetradeca-2E,4E,12Z-trien-8,10- } \\
\text { diynoic acid isobutylamide }\end{array}$ & Plasmodium falciparum & $26.7 \mu \mathrm{M}\left(\mathrm{IC}_{50}\right)$ & \\
\hline & & Root & $\begin{array}{l}\text { Tetradeca-2E,4E,12Z-trien-8,10- } \\
\text { diynoic acid isobutylamide }\end{array}$ & Trypanosoma brucei rhodesiense & $23.7 \mu \mathrm{M}\left(\mathrm{IC}_{50}\right)$ & \\
\hline & & Root & $\begin{array}{l}\text { Tetradeca-2E,4E,12Z-trien-8,10- } \\
\text { diynoic acid isobutylamide }\end{array}$ & Trypanosoma cruzi & $144 \mu \mathrm{M}\left(\mathrm{IC}_{50}\right)$ & \\
\hline & & Root & $\begin{array}{l}\text { Undeca-2E,4E-dien-8,10-diynoic } \\
\text { acid isopentylamide }\end{array}$ & Leishmania donovani & $16.6 \mu \mathrm{M}\left(\mathrm{IC}_{50}\right)$ & \\
\hline & & Root & $\begin{array}{l}\text { Undeca-2E,4E-dien-8,10-diynoic } \\
\text { acid isopentylamide }\end{array}$ & Plasmodium falciparum & $42.5 \mu \mathrm{M}\left(\mathrm{IC}_{50}\right)$ & \\
\hline & & Root & $\begin{array}{l}\text { Undeca-2E,4E-dien-8,10-diynoic } \\
\text { acid isopentylamide }\end{array}$ & Trypanosoma brucei rhodesiense & $18.9 \mu \mathrm{M}\left(\mathrm{IC}_{50}\right)$ & \\
\hline & & Root & $\begin{array}{l}\text { Undeca-2E,4E-dien-8,10-diynoic } \\
\text { acid isopentylamide }\end{array}$ & Trypanosoma cruzi & $66.9 \mu \mathrm{M}\left(\mathrm{IC}_{50}\right)$ & \\
\hline In vitro & Blood circulatory & Root & Pellitorine & $\begin{array}{l}\text { Caco-2 cell permeability (human colorectal } \\
\text { carcinoma) }\end{array}$ & $0.31 \mu \mathrm{g}$ & {$[37]$} \\
\hline In vitro & Immunomodulatory & Root & Aqueous & Spleen cell & $50 \mu \mathrm{g} / \mathrm{mL}$ & {$[4]$} \\
\hline In vitro & $\begin{array}{l}\text { Oxidative DNA } \\
\text { damage preventive }\end{array}$ & Root & Methanol (50\%) & $\begin{array}{l}\text { Fenton-induced damage of pBluescript II } \\
\text { SK (-) supercoiled DNA }\end{array}$ & $1.52 \mu \mathrm{g} / \mathrm{mL}$ & {$[33]$} \\
\hline
\end{tabular}

ABTS: 2,22'-azinobis (3-ethyl-benzothiazoline6-sulfonic acid); DPPH: 2,2-diphenyl-1-picrylhydrazyl; FRAP: Ferric Reducing Antioxidant Power; IC50: The half maximal inhibitory concentration; MIC: Minimum Inhibitory Concentration; MBC: Minimum Bactericidal Concentration; NS: Not stated; OH: Hydroxyl; TBARS: Thiobarburic acid reactive substances; Ref.: Reference. 


\subsubsection{Aphrodisiac activity}

In an investigation by Sharma et al., 50 and $100 \mathrm{mg} / \mathrm{kg}$ of root petroleum ether extract was orally administered to rats. This treatment elevated the precopulatory properties of male rats towards female rats. Also, there was an increase in penile erection index recognized, the mount was elevated four times, and intromission frequency was elevated three times [46].

\subsubsection{Blood circulatory activity}

Pellitorine $(5 \mathrm{mg} / \mathrm{mL})$ was isolated from roots was administered intracerebroventricularly and intravenously to rats. The outcomes revealed that pellitorine quickly and seamlessly permeated the gut mucosa and blood-brain barrier [37].

\subsubsection{Antihepatotoxic activity}

Usmani et al. investigated the antihepatotoxic properties of root ethanolic (50\%) extract (200 and $400 \mathrm{mg} / \mathrm{kg}$ ) in antitubercular drug-induced hepatotoxic rats. The results unveiled that the hepatic marker concentrations were reinstated and the root owns hepatoprotective action. Silymarin was utilized as a positive control in this investigation [48].

\subsubsection{Immunomodulatory activity}

A root aqueous extract at a dose of $10 \mathrm{mg} / \mathrm{kg}$ was orally administered to mice and it exhibited a better immunoenhancing index [4].

\subsubsection{Antineurotoxic activity}

Antineurotoxic effects of root aqueous and methanolic extracts (5 g/L) were researched in kainic acid-inducedstatus epileptic mice and it showed neuroprotective property against seizures encouraged by kainic acid [43].

\subsection{In vitro studies}

\subsubsection{Antibacterial activity}

The essential oil distilled from aerial parts applied on Staphylococcus aureus assay at a concentration of 1.25 $\mathrm{mg} / \mathrm{mL}$ unveiled antibacterial activity [21].

\subsubsection{Anticancer activity}

In a study by Mohammadi et al., ethanolic extract of aerial showed time-dependent anticancer effect against human colorectal cancer cells at IC $5064.75 \mu \mathrm{g} / \mathrm{mL}$ for 24 $\mathrm{h}$ and $\mathrm{IC}_{50} 105.9 \mu \mathrm{g} / \mathrm{mL}$ for $48 \mathrm{~h}$ [26].

\subsubsection{Antidementia activity}

Root ethanolic extract (IC50 $70 \mathrm{mg} / \mathrm{mL}$ ) inhibited acetylcholinesterase and Rivagistmine was used as a positive control in this investigation [27].

\subsubsection{Antidiabetic activity}

Kumar and Lalitha studied the antidiabetic effects of root ethanolic extract and it was noticed that at $\mathrm{IC}_{50} 29.25$ $\mu \mathrm{g} / \mathrm{mL}$ there was inhibition in the $\alpha$-amylase activity [29].

\subsubsection{Antifungal activity}

The essential oil of aerial showed antifungal activity against Candida albicans at a concentration of $0.72 \mathrm{mg} / \mathrm{mL}$ [21].

\subsubsection{Anti-inflammatory activity}

Deca-2E,4E-dienoic acid tyramide isolated from roots inhibited 5-lipoxygenase and cyclooxygenase distinctly at a concentration of $50 \mu \mathrm{g} / \mathrm{mL}$ [20].

\subsubsection{Antilipidemic activity}

In an investigation by Huerta et al., the aqueous extract inhibited rat intestinal disaccharidases (lipase). However, the authors did not state the part used and the concentration of the extract used in this investigation [28].

\subsubsection{Antimutagenic activity}

Root chloroform extract (1 $\mathrm{mg} /$ plate) generated inhibition in Ames Salmonella / microsome assay [32].

\subsubsection{Antioxidant activity}

An extract was prepared using root and methanol (50\%) exhibited antioxidant effects in peroxynitrite scavenging assay at $\mathrm{IC}_{50} 1.13 \mu \mathrm{g} / \mathrm{mL}$ [33].

\subsubsection{Antiprotozoal activity}

Dodeca-2E,4E-dienoic acid 4-hydroxy-2phenylethylamide $\left(\mathrm{IC}_{50} 5.97 \mu \mathrm{M}\right)$ was isolated from roots showed antiprotozoal activity in Trypanosoma cruzi assay [36].

\subsubsection{Blood circulatory activity}

Pellitorine also isolated from roots $(0.31 \mu \mathrm{g})$ can cross the Caco-2 cell monolayer from the apical-to-basolateral to basolateral-to-apical side [37].

\subsubsection{Immunomodulatory activity}

Bendjeddou et al. investigated the immunomodulatory effects of root aqueous extract in spleen cells. It was noticed that there was a better stimulation index at a concentration of $50 \mu \mathrm{g} / \mathrm{mL}$ [4].

\subsection{Toxicity studies}

There are three toxicity studies available regarding $A$. pyrethrum and they are discussed below.

Subchronic toxicity of root ethanolic extract (1000 $\mathrm{mg} / \mathrm{kg}$ ) was (orally administered) evaluated in rats for 90 days. This study revealed that there were no mortalities or adverse effects. Also, this extract had no treatmentassociated toxicological irregularities. Therefore, this study suggests the ethanolic extract is safe for chronic treatments [51].

In another study by Manouze et al., $5000 \mathrm{mg} / \mathrm{kg}$ of both aqueous and methanolic extracts (root) were separately orally administered to mice for 14 days. The results show that there was no toxicity-related 
symptoms, mortality, and weight changes in body and organs were observed after 14 days [30].

\section{Conclusion}

Several compounds have been isolated from $A$. pyrethrum and there are several ethnomedicinal uses of this plant species. However, only some scientific evidence is available in terms of bioactivities study. Therefore, more bioactivities and phytochemical linked researches should be conducted to generate more scientific evidence for the ethnomedicinal uses and identify more bioactive compounds that might be future lead compounds in drug discovery related researches. This work scrutinized, summed up, and recorded the currently available bioactive scientific evidence of $A$. pyrethrum.

\section{Acknowledgments}

This work received no funding. The authors are grateful to their family members for their support to deliver this work.

\section{References}

[1] Anacyclus pyrethrum (L.) Lag., Kewscience, 2020, http://powo.science.kew.org/taxon/urn:lsid:ipni.org:names:94136 6-1

[2] M. Adams, W. Alther, M. Kessler, M. Kluge, M. Hamburger, Malaria in the renaissance: Remedies from European herbals from the 16th and 17th century, J Ethnopharmacol, 133, 2011, 278-288.

[3] J. Bellakhdar, La Pharmacopée Marocaine Traditionnelle, 1997, France, Ibis Press.

[4] D. Bendjeddou, K. Lalaoui, D. Satta, Immunostimulating activity of the hot water-soluble polysaccharide extracts of Anacyclus pyrethrum, Alpinia galanga and Citrullus colocynthis, J Ethnopharmacol, 88, 2003, 155-160.

[5] R. Bentley, H. Trimen, Medicinal Plants, 2004, India, Asiatic Publishing House.

[6] L. Boulos, M. Gohary, Medicinal Plants of North Africa, 1983, U.S.A., Reference Publications Inc.

[7] P. Dioscorides, Kräuterbuch deß Uralten unnd in allerWelt berühmtesten Griechischen Scribenten Pedacii Dioscorides Anazarbaei, 1614, Germany, Corthoys.

[8] M. Grieve, A Modern Herbal. The Medical, Culinary, Cosmetic and Economic Properties, Cultivation and Folklore Modern Scientific Uses, 1989, UK, Jonathoan Cape.

[9] C. P. Khare, Indian Medicinal Plants: An Illustrated Dictionary, 2008, India, Springer Science \& Business Media.

[10] N. D. Prajapati, S. S. Purohit, A. K. Sharma, T. Kumar, Handbook of Medicinal Plants: A Complete Source Book, 2003, India, Agrobios.

[11] H. S. Puri, Rasayana: Ayurvedic Herbs for Longevity and Rejuvenation, 2002, UK, CRC Press.

[12] A. Singh, Compendia of World's Medicinal Flora, 2016, U.S.A., CRC Press.

[13] S. K. Sharma, M. Gupta, R. Bhadauria, S. Amanullah, H. C. Chandramoorthy, V. A. Kumar, S. Khatheeja, O. T. Asekun, D. S. Grierson, A. J. Afolayan, The Ayurvedic Pharmacopoeia of India,
Government of India, Ministry of Health and Family Welfare, 2004, India, Department of Indian System of Medicine and Homeopathy.

[14] S. V. Sathasivampillai, P. R. S. Rajamanoharan, M. Heinrich, Siddha Medicine in Eastern Sri Lanka Today-Continuity and Change in the Treatment of Diabetes, Front Pharmacol, 9, 2018, 1022.

[15] S. V. Sathasivampillai, P. R. S. Rajamanoharan, M. Munday, M. Heinrich, Plants used to treat diabetes in Sri Lankan Siddha Medicine - An ethnopharmacological review of historical and modern sources, J Ethnopharmacol, 198, 2017, 531-599.

[16] J. Boonen, V. Sharma, V. K. Dixit, C. Burvenich, B. D. Spiegeleer, LC-MS N-alkylamide profiling of an ethanolic Anacyclus pyrethrum root extract, Planta Med, 78, 2012, 1787-1795.

[17] K. Canli, A. Yetgin, I. Akata, E. M. Altuner, Antimicrobial activity and chemical composition screening of Anacyclus pyrethrum root, Indian J Pharm Educ, 51, 2017, S244-S248.

[18] Q.B. Chen, J. Gao, G.A. Zou, X.L. Xin, H. A. Aisa, Piperidine alkaloids with diverse skeletons from Anacyclus pyrethrum, J Nat Prod, 81, 2018, 1474-1482.

[19] S. Hamimed, N. Boulebda, H. Laouer, A. Belkhiri, Bioactivityguided isolation of alkamides from a cytotoxic fraction of the ethyl acetate extract of Anacyclus pyrethrum (L.) DC. roots, Curr Issues Pharm Medical Sci, 31, 2018, 180-185.

[20] B. Müller-Jakic, W. Breu, A. Pröbstle, K. Redl, H. Greger, R. Bauer, In vitro inhibition of cyclooxygenase and 5-lipoxygenase by alkamides from Echinacea and Achillea species', Planta Med, 60, 1994, 37-40.

[21] C. Selles, M. E. A. Dib, N. Djabou, F. Beddou, A. Muselli, B. Tabti, J. Costa, B. Hammouti, Antimicrobial activity and evolution of the composition of essential oil from Algerian Anacyclus pyrethrum L. through the vegetative cycle, Nat Prod Res, 27, 2013, 2231-2234.

[22] R. Ji, Q. Quan, X. Guo, J. Zhang, Y. Song, M. Zhu, P. Tan, J. Han, Y. Liu, Simultaneous determination of five $\mathrm{N}$-alkylamides in the root of Anacyclus pyrethrum by HPLC and profiling of components in its methanolic root extract by UPLC/Q-TOF-MS, Rev Bras Farmacogn, 29, 2019, 152-161.

[23] K. Muralikrishnan, S. Asokan, P. R. Geetha Priya, K. S. Zameer Ahmed, G, Ayyappadasan, Comparative evaluation of the local anesthetic activity of root extract of Anacyclus pyrethrum and its interaction at the site of injection in Guinea Pigs, Anesth Essays Res, 11, 2017, 444-448.

[24] V. K. Patel, R. V. Patel, H. Venkatakrishna-Bhatt, G. Gopalakrishna, G. Devasankariah, A clinical appraisal of Anacyclus pyrethrum root extract in dental patients, Phytother Res, 6, 1992, 158-159.

[25] N. Jalayer-Naderi, M. Niakan, E. Khodadadi, M. MohamadiMotlagh, The antibacterial activity of methanolic Anacyclus pyrethrum and Pistacia lentiscus L. extract on Escherichia coli, Iran J Microbiol, 8, 2016, 372-376.

[26] A. Mohammadi, B. Mansoori, P. C. Baradaran, S. C. Baradaran, B. Baradaran, Anacyclus pyrethrum extract exerts anticancer activities on the human colorectal cancer cell line (HCT) by targeting apoptosis, metastasis and cell cycle arrest, J Gastrointest Cancer, 48, 2017, 333-340

[27] K. Sujith, D. C. Ronald, V. Suba, Inhibitory effect of Anacyclus pyrethrum extract on acetylcholinesterase enzyme by in vitro methods, Pharmacogn J, 4, 2012, 48-51.

[28] V. Huerta, K. Mihalik, K. Beckett, V. Maitin, D. A. Vattem, Antidiabetic and anti-energy harvesting properties of common traditional herbs, spices and medicinal plants from India, J Nat Remedies, 2010, 10, 123-135.

[29] V. K. Kumar, K. G. Lalitha, In vitro study on $\alpha$-amylase inhibitory activity of an Ayurvedic medicinal plant, Anacyclus pyrethrum DC root, Indian J Pharmacol, 46, 2014, 350-351.

[30] H. Manouze, O. Bouchatta, A. C. Gadhi, M. Bennis, Z. Sokar, S. Ba-M'hamed, Anti-inflammatory, Antinociceptive, and 
Antioxidant activities of methanol and aqueous extracts of Anacyclus pyrethrum roots, Front Pharmacol, 8, 2017, 598.

[31] V. Rimbau, E. Risco, S. Canigueral, J. Iglesias, Anti-inflammatory activity of some extracts from plants used in the traditional medicine of North-African countries, Phytother Res, 10, 1996, 421423.

[32] K. Sukumaran, R. Kuttan, Inhibition of tobacco-induced mutagenesis by eugenol and plant extracts, Mutat Res-Genet Tox, 343, 1995, 25-30.

[33] M. D. Kalim, D. Bhattacharyya, A. Banerjee, S. Chattopadhyay, Oxidative DNA damage preventive activity and antioxidant potential of plants used in Unani system of medicine, BMC Complem Altern M, 10, 2010, 77.

[34] M. Riaz, M. Shahid, A. Jamil, M. Saqib, In vitro antioxidant potential of selected aphrodisiac medicinal plants, J Biol Reg Homeos Ag, 31, 2017, 419-424.

[35] D. K. Singh, S. B. Babbar, B. A. Mir, Studies on pellitorine production and antioxidant activity of explant specific calli of Anacyclus pyrethrum, J Biol Act Prod Nat, 10, 2020, 183-191.

[36] J. B. Althaus, C. Malyszek, M. Kaiser, R. Brun, T. J. Schmidt, Alkamides from Anacyclus pyrethrum L. and their in vitro antiprotozoal activity, Molecules, 22, 2017, 796.

[37] L. Veryser, N. Bracke, E. Wynendaele, T. Joshi, P. Tatke, L. Taevernier, B. De Spiegeleer, Quantitative in vitro and in vivo evaluation of intestinal and blood-brain barrier transport kinetics of the plant N-alkylamide pellitorine, Biomed Res Int, 2016, 5497402.

[38] V. Sharma, M. Thakur, N. S. Chauhan, V. K. Dixit, Evaluation of the anabolic, aphrodisiac and reproductive activity of Anacyclus pyrethrum DC in male rats, Sci Pharm, 77, 2009, 97-110.

[39] V. Sharma, J. Boonen, B. D. Spiegeleer, V. K. Dixit, Androgenic and spermatogenic activity of alkylamide-rich ethanol solution extract of Anacyclus pyrethrum DC, Phytother Res, 27, 2013, 99-106.

[40] K. Bezza, Z. El Gabbas, J. Laadraoui, M. A. Laaradia, S. Oufquir, A. Chait, Ameliorative potential of Anacyclus pyrethrum extract in generalized seizures in rat: Possible cholinergic mediated mechanism, Bangl J Pharmacol, 14, 2019, 188-195.

[41] D. Nirmala, Studies on anticonvulsant activity of Annacyclus pyrethrum in albino mice, Asian J Pharm Clin Res, 8, 2015, 178-187.

[42] M. Pahuja, J. Mehla, K. H. Reeta, S. Joshi, Y. K. Gupta, Root extract of Anacyclus pyrethrum ameliorates seizures, seizure-induced oxidative stress and cognitive impairment in experimental animals, Epilepsy Res, 98, 2012, 157-165.

[43] H. Manouze, O. Bouchatta, M. Bennis, Z. Sokar, S. Ba-M'hamed, Anticonvulsive and neuroprotective effects of aqueous and methanolic extracts of Anacyclus pyrethrum root in kainic acidinduced-status epilepticus in mice, Epilepsy Res, 158, 2019, 106225.

[44] S. R. Badhe, R. V. Badhe, M. M. Ghaisas, V. V. Chopade, A. D. Deshpande, Evaluations of antidepressant activity of Anacyclus pyrethrum root extract, Int J Green Pharm, 4, 2010, 79-82.

[45] M. Pahuja, J. Mehla, K. H. Reeta, M. Tripathi, Y. K. Gupta, Effect of Anacyclus pyrethrum on pentylenetetrazole-induced kindling, spatial memory, oxidative stress and rho-kinase II expression in mice, Neurochem Res, 38, 2013, 547-556.

[46] V. Sharma, M. Thakur, N. S. Chauhan, V. K. Dixit, Effects of petroleum ether extract of Anacyclus pyrethrum DC. on sexual behavior in male rats, Zhong Xi Yi Jie He Xue Bao, 8, 2010, 767773.

[47] D. Haghmorad, M. B. Mahmoudi, P. Haghighi, P. Alidadiani, E. Shahvazian, P. Tavasolian, M. Hosseini, M. Mahmoudi, Improvement of fertility parameters with Tribulus Terrestris and Anacyclus pyrethrum treatment in male rats, Int Braz J Urol, 45, 2019, 1043-1054.

[48] A. Usmani, M. Mujahid, M. Khushtar, H. H. Siddiqui, M. A. Rahman, Hepatoprotective effect of Anacyclus pyrethrum Linn. against antitubercular drug-induced hepatotoxicity in SD rats, J Complement Integr Med, 13, 2016, 295-300.
[49] F. Yousaf, M. Shahid, M. Riaz, A. Atta, H. Fatima, Immunomodulatory potential of Anacyclus pyrethrum (L.) and Mucuna pruriens (L.) in male albino rats, J Biol Regul Homeost Agents, 31, 2017, 425-429.

[50] V. Sharma, M. Thakur, N. S. Chauhan, V. K. Dixit, Immunomodulatory activity of petroleum ether extract of Anacyclus pyrethrum, Pharm Bio, 48, 2010, 1247-1254.

[51] K. Sujith, R. Darwin, V. Suba, Toxicological evaluation of ethanolic extract of Anacyclus pyrethrum in albino Wistar rats, Asian Pac J Trop Dis, 2, 2012, 437-441. 